\title{
Role of Tumour Markers CEA and CA19-9 in Colorectal Cancer
}

\author{
Rashmi Sreedhar ${ }^{1}$, Suhas Jajoo ${ }^{2}$, Meenakshi Yeola ${ }^{3}$, Yashwant Lamture ${ }^{4}$, Darshana Tote ${ }^{5}$ \\ 1, 2, 3, 4, 5 Department of General Surgery, Datta Meghe Institute of Medical Sciences, \\ Sawangi (Meghe), Wardha, Maharashtra, India.
}

Tumour markers Carcino-Embryonic Antigen (CEA) and carbohydrate antigen 19-9 (CA19-9) are used in the diagnosis of colorectal cancer. CEA levels are mainly used for the measurement of tumour response to therapy and for detecting recurrence of tumour. To study the use of CEA and CA 19-9 as tumour markers.

A total 32 studies has been included in this review till June 2020. A collective review of these studies regarding the use of CEA and CA 19-9, their statistical significance along with their limitations have been discussed and its conclusion is noted in this review article.

CEA and CA19-9 are useful in preoperative, postoperative, prognosis and follow up of the patients of colorectal cancer.

The two tumour markers mentioned above can be used preoperatively, postoperatively, after chemotherapy / radiotherapy and for prognostic significance in colorectal cancer. It can also be useful in many other adenocarcinomas like gastric adenocarcinoma, pancreatic adenocarcinoma, lung adenocarcinoma and ovarian adenocarcinoma.

\section{KEY WORDS}

CEA, CA19-9, Colorectal Cancer.
Corresponding Author: Dr. Rashmi Sreedhar, Flat No. C1, Honey Heights Apartments, Near SBI Bank, Sawangi (Meghe), Wardha - 442001,

Maharashtra, India.

E-mail: rashmis2993@gmail.com

DOI: $10.14260 /$ jemds $/ 2020 / 762$

How to Cite This Article:

Sreedhar R, Jajoo S, Yeola M, et al. Role of tumour markers CEA and CA19-9 in colorectal cancer. J Evolution Med Dent Sci 2020;9(46):3483-3488, DOI: $10.14260 /$ jemds $/ 2020 / 762$

Submission 08-08-2020,

Peer Review 01-10-2020,

Acceptance 08-10-2020,

Published 16-11-2020.

Copyright (C) 2020 Rashmi Sreedhar et al. This is an open access article distributed under Creative Commons Attribution License [Attribution 4.0 International (CC BY 4.0)] 


\section{BACKGROUND}

Carcinoembryonic antigen and carbohydrate antigen 19-9 are the tumour markers which are useful in the diagnosis of colorectal cancer. Carcinoembryonic antigen is an autoantigen present in the intestinal epithelia. It was discovered by Gold and Freedman in 1965. It is increased in colorectal cancers. ${ }^{1}$ CEA affects tumours by increasing the survival of tumour cells and angiogenesis of tumour. ${ }^{2}$ CEA is not useful in the screening of colorectal cancer. Preoperative CEA levels are helpful in staging and planning the surgical management of patients with colorectal carcinoma. Preoperative elevation of CEA levels indicate poor prognosis, CEA is used for monitoring during systemic therapy especially in cases of metastatic colorectal cancer. $^{3}$ Thus, serum CEA levels are used to monitor recurrence of the cancer after surgery, but is not helpful in screening of the disease. ${ }^{4}$

Carbohydrate antigen 19-9 is a type of glycosphingolipid. Serum CA 19-9 levels are mostly increased in patients with gastrointestinal cancers, such as pancreatic, colon, rectal, stomach, and liver cancers. ${ }^{5}$ CA $19-9$ has been used in clinical practice along with CEA as a tumour marker. CA 19-9 have been useful for finding the prognostic significance of the disease and for monitoring the recurrence of the disease. ${ }^{6}$ It is a tumour marker that is observed in elevated serum concentration with metastatic colon cancer. ${ }^{7}$

Preoperative CEA is indicated on all newly diagnosed colorectal cancer, normalization after surgical resection is expected and serial assays to be monitored on follow up visits. ${ }^{8}$ Routine serum CEA levels are helpful to monitor the response of the patient to therapy, when elevated CEA levels normalize after total tumour resection, or during chemotherapy or radiotherapy, it indicates good response to therapy. Serial CEA levels are helpful in the early detection of tumour recurrence in patients who have undergone resection; as these levels are elevated in distant metastases as well as in local recurrences. The appearance of elevated serum levels of these markers is a sign of recurrence or metastatic lesions in near environment of the tumour as well as remote ones. ${ }^{7}$ Raised CEA levels can also be used as a basis for a 'second-look' surgery. ${ }^{9}$

Moreover, combined detection of CEA and CA 19-9 is more sensitive than separate detection in evaluating CRC (ColoRectal Cancer) prognosis. ${ }^{10}$ Thus, in my study I have considered the role of tumour markers CEA and CA 19-9.

\section{METHODS}

\section{Search Strategy}

A comprehensive set of keywords or search terms such as CEA, CA 19-9, tumour markers, colorectal cancer were selected. We searched PubMed, Scopus, Google scholar and Cochrane databases. Relevant observational studies were selected till July 2020. The list of reference articles were retrieved and were searched via automation and manually. Only human studies and English language studies were selected.

\section{Data Extraction}

Data was extracted by 2 authors into separate forms on Microsoft excel 2020. Article selection and data extraction discrepancies were resolved through discussion. No publication date restriction was set. Automated and manual reduplications were performed.

\section{Inclusion Criteria}

Those research studies were selected which assessed the use of CEA and CA 19-9 tumour markers in colorectal cancer, use of the tumour markers CEA and CA 19-9 in other cancers.

\section{Exclusion Criteria}

Studies which assessed the use of tumour markers other than CEA and CA 19-9 in colorectal cancer and tumour markers other than CEA and CA 19-9 in malignancies other than colorectal cancer.

\section{DISCUSSION}

Tumour markers currently used in colorectal malignancies are serum CEA and CA 19-9 levels. CEA can be ordered preoperatively in patients with CRC as it would assist in staging \& surgical treatment planning. Preoperative elevated levels is a poor prognostic marker. If the patient of a stage II or stage III disease is a candidate for surgery or systemic therapy, then postoperatively CEA levels should be done every 3 months, for at least 3 years after diagnosis. Postoperative CEA shows whether the resection is complete or not. It is also useful in follow up visits of the patients. It also helps in monitoring the response to chemotherapy and radiotherapy. It is the biomarker of choice for monitoring metastatic disease during systemic therapy as indicated by 2 studies. CEA is a useful biomarker in assessing the recurrence in 3 studies. It is also an indicator of survival rate of CRC patients in 2 studies. It is more useful in colorectal adenocarcinoma.

Sensitivity of CEA was as high as $90 \%$ in 1 study but other studies less sensitivity was observed but the specificity of CEA was more than $90 \%$ in all of the studies. CA 19-9 is also used as a tumour biomarker but there is less evidence to suggest its use in clinical practice. Sensitivity was about $50 \%$ as suggested in 2 studies and specificity was a little higher than $50 \%$ as seen in 3 studies. There is 1 study which showed similar results as CEA in suggesting poor prognosis. It is also been found in a study that the sensitivity and specificity increase when both CEA and CA 19-9 were combined. Many other markers like KRAS and BRAF mutations are useful for assessing response of anti EGFR (Epidermal Growth Factor Receptor) therapy and prognostic stratification in colorectal cancer. Many other tumour biomarkers such as p53, RAS, MSI (Micro-Satellite Instability) have been tried but are not recommended in colorectal cancer.

\section{Review of Previous Studies}

J.Y. Kim et al serum CEA levels were done at different time points, 1 preoperative value and 2 postoperative values of CEA on postoperative day number 7 and postoperative day number 30. 


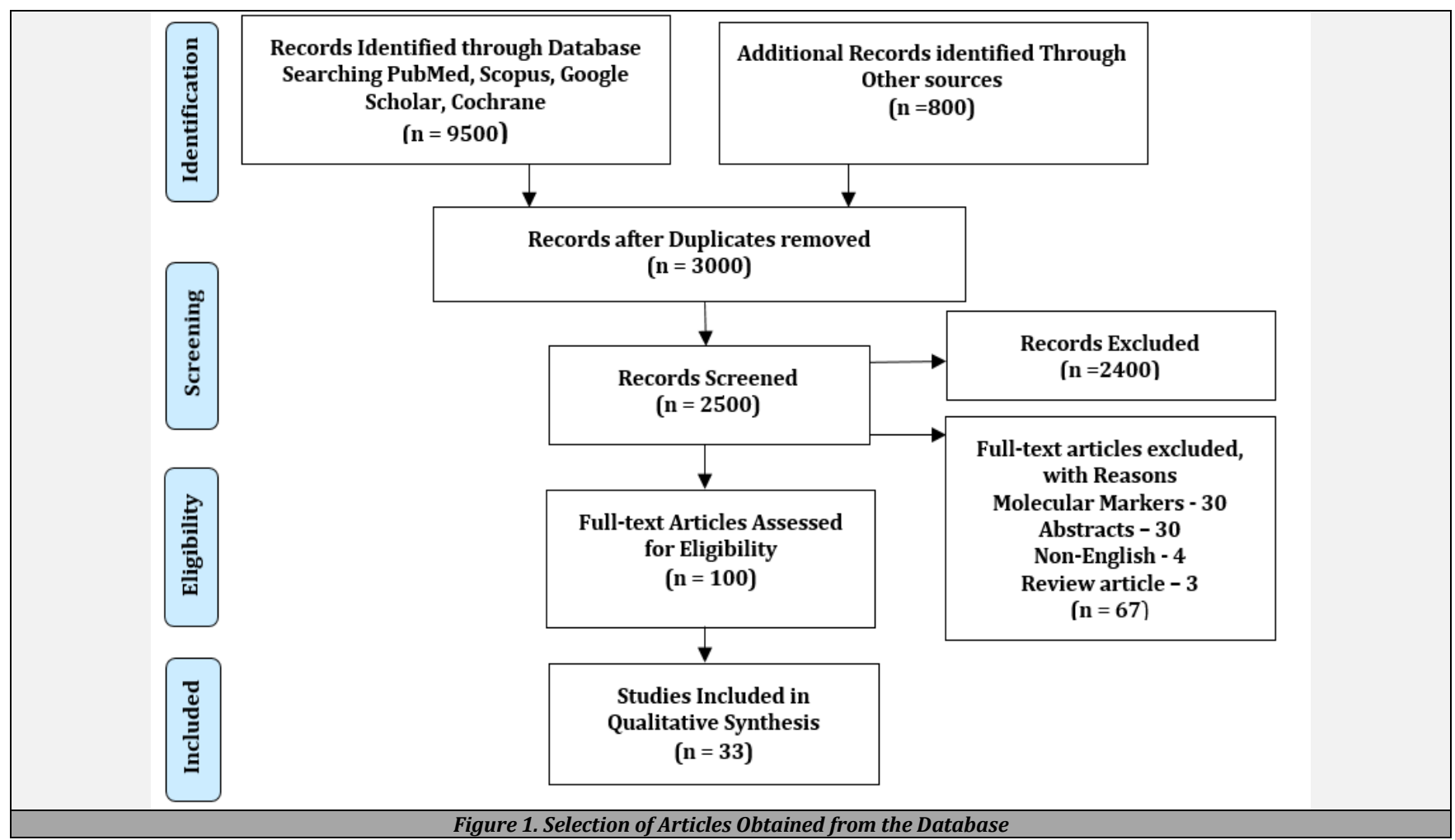

The CEA levels on postoperative day 7 showed significant overall survival rate but did not have any significance in disease free survival rate and the CEA levels on postoperative day 30 was related significantly to both overall survival and disease-free survival. A single postoperative CEA value have decreased value in detecting the change in tumour biology.11

In Hayder et al carcinoma cases showed no false positives and $10.0 \%$ false negatives for CEA levels. Sensitivity of CEA in detecting colorectal cancer was $90 \%$ and specificity of CEA in detecting colorectal carcinoma is $100 \%$ respectively. Carcinoma cases showed no false positives for CA 19-9, with $70.0 \%$ false negatives. Sensitivity of CA 19-9 in detecting colorectal cancer was $30 \%$ and specificity of CA 19-9 was found to be $100 \%$. If the above mentioned markers are elevated in the serum it is a suggestive of recurrence of the disease or presence of metastasis. There was positive correlation of CEA in colorectal carcinomas. ${ }^{12}$

Polat et al. At the cut-off level of $4.8 \mathrm{ng} / \mathrm{mL}$, sensitivity of CEA for detecting colorectal carcinoma was $43.9 \%$ and the specificity of CEA for detecting colorectal carcinoma was 95 $\% .^{13}$

Zora et al Serum CEA and CA 19-9 levels were increased in the patients aged $56-65$ years in $31.9 \%$ of cases and in the patients of age 66 - 75 years in $36.3 \%$ of cases. $37.4 \%$ of cases were localized in the rectum, followed by sigmoid colon with $27.5 \%$ of cases and only $2.2 \%$ of cases were in caecum and ascending colon. ${ }^{7}$

\section{Type of Tumours}

Adenocarcinoma in $97.8 \%$ cases and mere $1.1 \%$ of cases of intraepithelial and squamous cell carcinoma type. Metastases were observed in $40.7 \%$ of cases, predominantly in lymph nodes in $35.2 \%$ of cases, metastases in the liver in $24.2 \%$ of cases, metastasis to the lungs in $5.5 \%$ and $1 \%$ in the adipose tissue.
Imran Bhatti et al showed that sensitivity of CEA in cases of recurrence was $83 \%$ and specificity of the same was $95 \%$. The CEA levels were more increased in cases of recurrence (true positives) with average CEA of $34 \mathrm{ng} / \mathrm{ml}$, than those without recurrence (false positives) who had average CEA of 7 $\mathrm{ng} / \mathrm{ml}$. Greater the preoperative levels of CEA greater the risk of developing recurrent disease and poor prognosis. After complete resection of the tumour, CEA levels fall and normal levels are reached within 4 to 6 weeks. Elevated CEA levels following surgery indicates incomplete resection or occult metastasis. ${ }^{14}$

Louhimo et al showed that in cases of colorectal cancer the elevated preoperative CEA levels \& CA 19-9 levels indicate poor outcome in patients with colorectal carcinoma. Tumour marker CEA is also an independent prognostic factor. ${ }^{15}$

Carpelan-Holmstrom et al. also observed CEA elevation is more in patients with rectal carcinoma as compared to CEA levels seen in patients with colon carcinoma. ${ }^{16}$

Bombski et al showed that CEA levels were higher in cases with distal tumours $(9.1 \pm 1.1 \mathrm{ng} / \mathrm{mL})$ in comparison to cases with proximal tumours $(1.48 \pm 0.1 \mathrm{ng} / \mathrm{mL})$. CA 19-9 levels showed similar results as CEA. They were higher in cases with distal tumours $(19.9 \pm 2.1 \mathrm{U} / \mathrm{mL})$ in comparison to those with proximal tumours $(1.8 \pm 0.2 \mathrm{U} / \mathrm{mL}) .{ }^{17}$

Hamada et al showed that CEA levels were elevated in patients of colon cancer with lymphatic and vascular invasion in the primary tumour. ${ }^{18}$

Khoo et al. showed that the CEA levels of metastatic colon cancer is grossly increased in metastatic regions as compared the CEA levels of the primary tumour. ${ }^{19}$

Filella and colleagues compared CEA and CA 19-9 levels in diagnosed colorectal carcinoma patients who came for follow up. CEA levels were abnormal in $84 \%$ with recurrent disease and CA 19-9 levels were abnormal in only $48 \%$. CA 19-9 results gave no additional benefit in planning the management 
of colorectal carcinoma compared to information provided by CEA. ${ }^{20}$

Slentz et al. demonstrated that five year survival rate is poor in patients with higher preoperative CEA levels compared to those with a normal preoperative CEA level. They concluded that preoperatively elevated CEA, is a poor prognostic factors especially in patients where the CEA levels does not normalize after a curative resection for colorectal cancer. ${ }^{21}$

Korenaga et al. provided further evidence that CEA levels as a prognostic tool in recurrent gastrointestinal malignancies. Patients who had higher CEA levels with colorectal cancer recurrence as seen during follow-up after curative resection were considered. The doubling time of serum CEA levels was examined as an index of tumour growth. It was concluded that CEA doubling time was a major independent prognostic factor in colorectal cancer patients. ${ }^{22}$

Moertel et al. CEA testing for cancer recurrence and in deciding the interventions (diagnostic \& surgical) and the end result in a cohort of 1,216 patients following curative resection of CRC. It was concluded that cancer cures attributable to surgery performed on the basis of CEA monitoring were, at best, infrequent. ${ }^{23}$

Pietra et al. showed that the effectiveness of follow-up CEA levels were evaluated based on detection and resectability of recurrent disease and survival after surgery in colorectal carcinoma patients. Pietra and colleagues concluded that CEA levels were a very good indicator of recurrent disease and frequent CEA levels are useful in the follow-up program. ${ }^{24}$

De Hass et al. showed the similarities between CT (Computed Tomography) scan and CEA levels in $>90 \%$ of cases. The radiological response (stable disease) was similar to CEA response in $94 \%$ of cases, while in patients with radiological evidence (disease progression) was similar to CEA response in $95 \%$ of cases. CT and CEA both are equally effective in assessing response of hepatic metastasis to chemotherapy in a case of colorectal cancer. ${ }^{25}$

Won Kyung Cho et al showed that serum CEA levels after treatment detects recurrence of the disease and the prognosis after recurrence of the disease. Serum CEA monitoring should be done in tumours with normal pre-operative CEA levels. One-fourth of tumours have normal CEA levels initially but when recurrent disease occurs there is an elevated CEA level. ${ }^{26}$

$C$ Aggarwal et al showed that metastatic colorectal cancer patients undergoing chemotherapy who have elevated CEA levels have decreased survival rate. CEA levels are independent prognostic markers for patients undergoing chemotherapy for metastatic disease. ${ }^{27}$

Y. Takahashi et al showed that preoperative abnormal levels for CEA, and CA 19-9, and both combined were $28.3 \%$, $29.2 \%$, and $45.0 \%$, respectively. With gastric cancers, the sensitivities for recurrence were $65.8 \%, 55 \%$ and $85 \% .^{28}$

J Lundin et al showed that survival of patients with pancreatic cancer varies with a low vs. high preoperative tumour marker level, if stage of disease is not taken into consideration. When stage was taken into consideration, the variation was significant only in stage II and stage III, in patients with resectable disease and those without resectable disease, and only for CA 19-9. The difference in survival reflects the difference in tumours biological behaviour with a low CA 19-9 level in contrast to a high CA 19-9 level. ${ }^{29}$
Wang et al showed that there is significant difference of serum CEA levels in patients with lung cancer in presence or absence of tumour metastasis and the effectiveness of CEA levels to determine tumour metastasis was studied. The sensitivity of serum CEA level was $85.1 \%$ and specificity of serum CEA level was $59.7 \%{ }^{30}$

Lin et al showed that preoperative serum CEA levels were higher in cases of advanced stage of primary mucinous ovarian carcinoma and indicates a poor prognosis. ${ }^{31}$

N. R. Hall et al showed that sensitivity and specificity of CEA alone is $76 \& 86 \%$ respectively and that of CA 242 is 60 \& $87 \%$ respectively. When both were combined the sensitivity and specificity were $48 \%$ and $95 \%$ when both are abnormal and $88 \%$ and $78 \%$ when either is abnormal. ${ }^{32}$

Nasif et al showed that when serum CEA and p53 both were combined in CRC they showed a more statistically significant results in terms of clinical outcome, mean survival rate and shows the disease progression from an in situ cancer to invasive cancer than both separately. ${ }^{33}$

\section{CONCLUSIONS}

CEA and CA 19-9 are done in preoperative, postoperative and metastatic setting. CEA done preoperatively is used for staging of the disease and planning the surgical intervention. Raised preoperative CEA levels ( $>5 \mathrm{ng} / \mathrm{mL}$ ) are associated with poor prognosis. Early cancer and increase in stage show raised preoperative CEA levels. Elevated preoperative CEA levels in colorectal carcinoma patients in stage III and stage IV is a risk factor for local recurrence and also for decrease in disease free survival and overall survival.

CEA levels usage for postoperative surveillance should be assessed before resection. Preoperative raised CEA levels is an indicator as to whether they would be useful for surveillance or not. Post-operatively, CEA levels should be done 3 monthly in colorectal carcinoma patients which is of stage II and stage III disease for 3 years, if the patient has a disease which is resectable or is a candidate for systemic therapy. Raised CEA levels should be evaluated in metastatic disease, but there is no information regarding whether to give adjuvant therapy or systemic therapy for the metastasis.

CEA levels are important indicators for recurrence in asymptomatic patients, is cost effective than radiology for detecting recurrence and liver metastases. In patients with metastatic colorectal cancer, CEA levels are the tumour markers of choice during systemic therapy for disease monitoring. CEA levels must be measured before treatment for metastatic disease and repeated 1 to 3 monthly during antiblastic treatment. Persistently elevated values above baseline, restaging must be done and suggests progressive disease with no radiographic evidence. There may be elevated CEA levels in the initial 4 to 6 weeks of a new anti-blastic treatment, especially after usage of some drugs ex: oxaliplatin.

The usage of combination of tumour markers aids in the diagnosis of colorectal cancer prognosis. Few combinations of CEA with CA 242 and with p53 have shown significant results. CEA and CA 19-9 levels are useful for detecting recurrence in patients with gastric cancers. It is useful in predicting overall survival of patients diagnosed with pancreatic cancers. CEA is also a useful prognostic marker in ovarian and lung cancer. 


\section{Abbreviations}

- $\quad$ CEA - Carcino-Embryonic Antigen

- $\quad$ CA 19-9 - Carbohydrate Antigen 19-9.

- CRC - Colo-Rectal Cancer.

Financial or other competing interests: None.

Disclosure forms provided by the authors are available with the full text of this article at jemds.com.

\section{REFERENCES}

[1] Scurr MJ, Brown CM, Costa Bento DF, et al. Assessing the prognostic value of preoperative carcinoembryonic antigen-specific T-cell responses in colorectal cancer. J Natl Cancer Inst 2015;107(4):djv001.

[2] Prager GW, Braemswig KH, Martel A, et al. Baseline carcinoembryonic antigen (CEA) serum levels predict bevacizumab-based treatment response in metastatic colorectal cancer. Cancer Sci 2014;105(8):996-1001.

[3] Locker GY, Hamilton S, Harris J, et al. ASCO 2006 update of recommendations for the use of tumor markers in gastrointestinal cancer. J Clin Oncol 2006;24(33):531327.

[4] Wu CW, Sung JJY. Colorectal cancer screening: are stool and blood based tests good enough. Chin Clin Oncol 2013;2(1):8.

[5] Bagaria B, Sood S, Sharma R, et al. Comparative study of CEA and CA 19-9 in esophageal, gastric and colon cancers individually and in combination (ROC curve analysis). Cancer Biol Med 2013;10(3):148-57.

[6] Sato H, Usuda N, Kuroda M, et al. Significance of serum concentrations of E-selectin and CA 19-9 in the prognosis of colorectal cancer. Jpn J Clin Oncol 2010;40(11):107380.

[7] Vukobrat-Bijedic Z, Husic-Selimovic A, Sofic A, et al. Cancer antigens (cea and ca 19-9) as markers of advanced stage of colorectal carcinoma. Med Arch 2013;67(6):397401.

[8] Recio-Boiles A, Waheed A, Cagir B. Cancer colon. Treasure Island (FL): StatPearls Publishing 2020.

[9] Treasure T, Monson K, Fiorentino F, et al. The CEA secondlook trial: a randomised controlled trial of carcinoembryonic antigen prompted reoperation for recurrent colorectal cancer. BMJ Open 2014;4(5):e004385.

[10] Xu X, Niu M, Zhang B, et al. Clinical value of CEA and CA 19-9 in colorectal cancer by Kaplan-Meier survival curve. Int J Clin Exp Med 2019;12(12):13305-10.

[11] Kim JY, Kim NK, Sohn SK, et al. Prognostic value of postoperative CEA clearance in rectal cancer patients with high preoperative CEA levels. Ann Surg Oncol 2009;16(10):2771-8.

[12] Azzal HS, Al-Wasiti EAR, Qasim B. Serum CEA and CA 199 along the colorectal adenoma - carcinoma sequence. Int J Adv Res 2015;3(12):1628-35.

[13] Polat E, Duman U, Duman M, et al. Diagnostic value of preoperative serum carcinoembryonic antigen and carbohydrate antigen $19-9$ in colorectal cancer. Curr Oncol 2014;21(1):e1-7.

[14] Bhatti I, Patel M, Dennison AR, et al. Utility of postoperative CEA for surveillance of recurrence after resection of primary colorectal cancer. Int J Surg 2015;16(pt A):123-8.

[15] Louhimo J, Carpelan-Holmstrom M, Alfthan H, et al. Serum HCG beta, CA 72-4 and CEA are independent prognostic factors in colorectal cancer. Int J Cancer 2002;101(6):5458.

[16] Carpelan-Holmstrom MA, Haglund $\mathrm{CH}$, Roberts PJ. Difference in serum tumor markers between colon and rectal cancer. Dis Colon Rectum 1996;39(7):799-805.

[17] Bombski G, Gasiorowska A, Orszulak-Michalak D, et al. Differences in plasma gastrin, CEA, and CA 19-9 concentration in patients with proximal and distal colorectal cancer. Int J Gastrointest Cancer 2002;31(13):155-63.

[18] Hamada Y, Yamamura M, Hioki $K$, et al Immunohistochemical study of carcinoembryonic antigen in patients with colorectal cancer. Correlation with plasma carcinoembryonic antigen levels. Cancer 1985;55(1):136-41.

[19] Khoo SK, Warner NL, Lie JT, et al. Carcinoembryonic antigenic activity of tissue extracts: a quantitative study of malignant and benign neoplasms, cirrhotic liver, normal adult and fetal organs. Int J Cancer 1973;11(3):681-7.

[20] Filella X, Molina R, Pique JM, et al. Use of CA 19-9 in the early detection of recurrences in colorectal cancer: comparison with CEA. Tumor Biol 1994;15(1):1-6.

[21] Slentz K, Senagore A, Hibbert J, et al. Can preoperative and postoperative CEA predict survival after colon cancer resection? Am Surg 1994;60(7):528-31.

[22] Korenaga D, Saeki H, Mawatari K, et al. Serum carcinoembryonic antigen concentration doubling time correlates with tumor biology and life expectancy in patients with recurrent gastrointestinal carcinoma. Arch Surg 1997;132(2):188-94.

[23] Moertel CG, Fleming TR, Macdonald JS, et al. An evaluation of the carcinoembryonic antigen (CEA) test for monitoring patients with resected colon cancer. JAMA 1993;270(8):943-7.

[24] Pietra N, Sarli L, Costi R, et al. Role of follow-up in management of local recurrences of colorectal cancer: a prospective, randomized study. Dis Colon Rectum 1998;41(9):1127-33.

[25] de Haas RJ, Wicherts DA, Flores E, et al. Tumor marker evolution: comparison with imaging for assessment of response to chemotherapy in patients with colorectal liver metastases. Ann Surg Oncol 2010;17(4):1010-23.

[26] Cho WK, Choi DH, Park HC, et al. Elevated CEA is associated with worse survival in recurrent rectal cancer. Oncotarget 2017;8(62):105936-41.

[27] Aggarwal C, Meropol NJ, Punt CJ, et al. Relationship among circulating tumor cells, CEA and overall survival in patients with metastatic colorectal cancer. Ann Oncol 2013;24(2):420-8.

[28] Takahashi Y, Takeuchi T, Sakamoto J, et al. The usefulness of CEA and/or CA19-9 in monitoring for recurrence in gastric cancer patients: a prospective clinical study. Gastric Cancer 2003;6(3):142-5.

[29] Lundin J, Roberts PJ, Kuusela P, et al. The prognostic value of preoperative serum levels of CA 19-9 and CEA in patients with pancreatic cancer. $\mathrm{Br} \mathrm{J}$ Cancer 1994;69(3):515-9. 
[30] Wang J, Chu Y, Li J, et al. The clinical value of carcinoembryonic antigen for tumor metastasis assessment in lung cancer. PeerJ 2019;7:e7433.

[31] Lin W, Cao D, Shen K. Prognostic significance of preoperative serum CEA in primary mucinous ovarian carcinoma: a retrospective cohort study. Cancer Manag Res 2018;10:6913-20.
[32] Hall NR, Finan PJ, Stephenson BM, et al. The role of CA-242 and CEA in surveillance following curative resection for colorectal cancer. Br J Cancer 1994;70(3):549-53.

[33] Nasif WA, Lotfy M, El-Sayed IH, et al. Implications of CEA and p53 overexpression in the poor prognosis of colorectal cancer. Med Oncol 2006;23(2):237-44. 